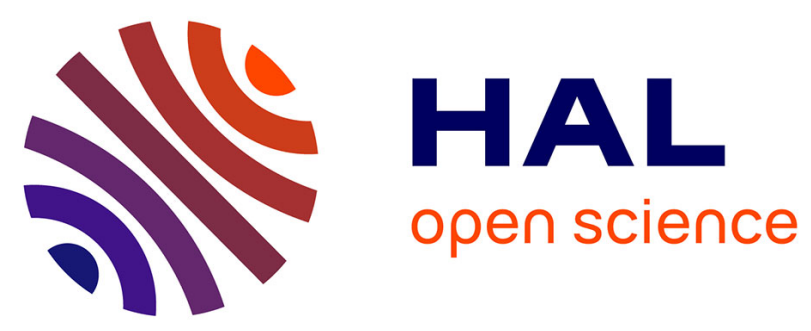

\title{
Differential expression of cyclic nucleotide phosphodiesterases 4 in developing rat lung.
}

Emmanuel Lopez, Pierre-Henri Jarreau, Elodie Zana, Marie-Laure

Franco-Montoya, Thomas Schmitz, Danièle Evain-Brion, Jacques R. Bourbon, Christophe Delacourt, Céline Méhats

\section{To cite this version:}

Emmanuel Lopez, Pierre-Henri Jarreau, Elodie Zana, Marie-Laure Franco-Montoya, Thomas Schmitz, et al.. Differential expression of cyclic nucleotide phosphodiesterases 4 in developing rat lung.. Developmental Dynamics, 2010, 239 (9), pp.2470-8. 10.1002/dvdy.22374 . hal-00526901

\section{HAL Id: hal-00526901 https://hal.science/hal-00526901}

Submitted on 16 Oct 2010

HAL is a multi-disciplinary open access archive for the deposit and dissemination of scientific research documents, whether they are published or not. The documents may come from teaching and research institutions in France or abroad, or from public or private research centers.
L'archive ouverte pluridisciplinaire HAL, est destinée au dépôt et à la diffusion de documents scientifiques de niveau recherche, publiés ou non, émanant des établissements d'enseignement et de recherche français ou étrangers, des laboratoires publics ou privés. 
Differential Expression of Cyclic Nucleotide Phosphodiesterases 4 in Developing Rat Lung

Running Title: PDE4 in developing rat lung

Emmanuel Lopez ${ }^{1,2,3,4}$, Pierre-Henri Jarreau ${ }^{1,2,3,4}$, Elodie Zana ${ }^{1,3}$, Marie-Laure Franco-

Montoya $^{3,5}$, Thomas Schmitz ${ }^{1,2,3,6}$, Danièle Evain-Brion ${ }^{1,3}$, Jacques Bourbon ${ }^{3,5}$, Christophe

Delacourt $^{3,5}$, Céline Méhats ${ }^{1,3, *}$

${ }^{1}$ UMR 767 Inserm-Paris Descartes, Paris, France; ${ }^{2}$ Université Paris Descartes, Faculté de Médecine, Paris, France; ${ }^{3}$ PremUP, Paris, France; ${ }^{4}$ AP-HP, Groupe hospitalier Cochin-Saint Vincent de Paul, Service de Médecine Néonatale de Port-Royal, Paris, France; ${ }^{5}$ Inserm U955, Créteil, France; ${ }^{6}$ AP-HP, Groupe hospitalier Cochin-Saint Vincent de Paul, Service d'Obstétrique, Maternité de Port-Royal, Paris, France.

* Corresponding author: Céline Méhats, UMR 767 Inserm-Paris Descartes, Hôpital Saint Vincent de Paul, 82 Avenue Denfert-Rochereau, 75014 Paris, France.

E-mail address: celine.mehats@inserm.fr

Phone: 331404882 38, Fax: 33140488394

Statement of financial support: This work was supported by grants from la Chancellerie des Universités de Paris (Legs Poix 2006) and from Air Liquide Foundation. 


\begin{abstract}
During the perinatal period, lungs undergo changes to adapt to air breathing. The genes involved in these changes are developmentally regulated by various signaling pathways, including the cyclic nucleotide cAMP. As PDE4s are critical enzymes for regulation of cAMP levels, the objective of this study was to investigate PDE4s ontogeny in developing rat lung during the perinatal period. Pulmonary PDE4 activity, PDE4A-D and PDE4B and PDE4D variants expression levels, PDE4B and PDE4D protein levels, and PDE4Ds localization in distal lung were determined. PDE4 activity increased towards term, dropped at birth, and increased thereafter to reach a plateau at the end of the second week of life. PDE4B2 and PDE4D long forms demonstrated a pattern of expression that increased markedly at birth. After birth, PDE4D were expressed in alveolar epithelial and mesenchymal cells. The study therefore evidenced striking variations in expression patterns among the PDE4 family that differed from changes in global PDE4 activity.
\end{abstract}

Key words: lung development, phosphodiesterases 4, cyclic AMP 


\section{Introduction}

Successful transition to air breathing at birth involves dramatic changes in lung physiology, including differentiation of alveolar type II cells, clearance of pulmonary fluid, decrease of pulmonary vascular resistance, and surfactant release (Cardoso, 2001). Defects in these physiological changes may affect neonates and can be responsible for neonatal respiratory disorders, which represent the leading cause of hospitalization in neonatal intensive care units. Preterm infants may develop bronchopulmonary dysplasia (BPD), a disease mainly related to impairment of alveolarization and microvascular development. Identification of the molecular and physiological processes controlling perinatal lung development and alveolarization would be promising to explore and develop new therapeutic approaches to cure or prevent respiratory disorders.

Lung adaptation to air breathing and lung development are controlled by a large number of factors. Many of these factors involve signaling pathways involving cAMP (Mendelson, 2000). Earlier studies have focused on developmental changes in cAMP/PKA pathway as key controllers of the actions of catecholamines or other hormones in perinatal lungs.

Manipulation of the cAMP pathway facilitated surfactant release and reduced surface tension in experimental models (Aeberhard et al., 1984; Odom et al., 1987; Fisher et al., 1991). Ontogenic variations in PKA and some of its protein substrates have been described: the phosphorylation pattern and the expression of specific phosphatase or adenylate cyclase activities changed during lung development (Whitsett et al., 1982a; Whitsett et al., 1982b; Whitsett et al., 1983; Whitsett et al., 1985; Acarregui et al., 1998).

Cyclic nucleotide phosphodiesterases (PDE) represent a superfamily of enzymes that hydrolyze cAMP. Eleven families of PDE have been described in mammals, and selective inhibitors of specific families are now used or proposed to treat various disorders including cardiovascular disease, erectile dysfunction, pulmonary arterial hypertension, and chronic 
pulmonary disorders (Bender and Beavo, 2006). Among these families, the PDE4 family has been extensively investigated over recent decades for its potential as a new pharmacological target in asthma and chronic obstructive pulmonary disease in adults (Boswell-Smith and Spina, 2007; Spina, 2008).

PDE4s are encoded by four genes designated A through D, and encompass more than 20 different proteins produced through differential promoter activation (Conti et al., 2003). Because of this diversity, each PDE4 protein is now thought to have a specific, non-redundant function in controlling cAMP metabolism within physiological processes (Conti et al., 2003). The ontogeny and precise role of PDE4s in the perinatal developing lung and alveolarization have not yet been investigated.

Rat pups are a common experimental model to study normal or pathological distal lung development. They are born at the saccular phase of lung development and alveolarization is an entirely postnatal event, starting on the fourth postnatal day and ending at day 21. The potential of PDE4 inhibition was evaluated in an experimental model of BPD in rat pups and demonstrated that PDE4 inhibition may impair alveolarization (Mehats et al., 2008). Although a global impact via a decrease of somatic growth is certainly involved in this deleterious effect, the possibility of a specific involvement of PDE4 proteins in lung development must also be considered.

The present study investigated global PDE4 activity and specific PDE4 protein expression patterns in fetal and newborn rats. Specific isoforms of PDE4B and PDE4D varied profoundly during distal lung development, suggesting a role for these proteins in perinatal lung physiology. 


\section{Results}

\section{PDE4 activity in lung during fetal and early postnatal life}

At fetal day 17 (F17, term F22), PDE4 activity was 14 \pm 2 pmol/min/mg and increased significantly towards term, doubling at F21 (28 $\pm 3 \mathrm{pmol} / \mathrm{min} / \mathrm{mg})$ (Figure 1). After birth, PDE4 activity decreased transiently $(16 \pm 2 \mathrm{pmol} / \mathrm{min} / \mathrm{mg})$ before increasing sharply from P7

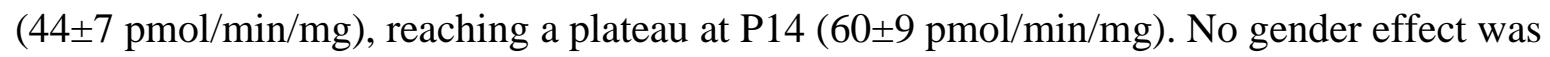
observed (at P1 males: $18 \pm 1 \mathrm{pmol} / \mathrm{min} / \mathrm{mg}, \mathrm{n}=5$; females: $17 \pm 4 \mathrm{pmol} / \mathrm{min} / \mathrm{mg}, \mathrm{n}=6$; other data not shown).

\section{Pulmonary PDE4 mRNA levels during fetal and early postnatal life}

Developmental changes in mRNA levels of the four PDE4 genes were investigated using generic primers covering all known variants derived from each gene. Data are expressed as $\Delta \mathrm{Ct}$, which means that a variation of 1 cycle represents a 2 -fold increase or decrease and that a low $\triangle \mathrm{Ct}$ indicates high expression. At F17, PDE4A and PDE4B mRNAs were the most abundant forms, with 8-fold lower and 1,000-fold lower expression of PDE4D and PDE4C, respectively (Table 2). PDE4C remained the PDE4 with the lowest expression at all stages examined, although PDE4C mRNA increased significantly after F21 (Figure 2). PDE4A mRNA expression levels did not change significantly either during saccular and alveolar stages, or at birth. PDE4B and PDE4D mRNAs rose at F21, peaked on P1 to reach 25- and 60-fold their F17 levels, respectively, then decreased 2- to 3-fold at the beginning of alveolarization and decreased further at P21 (Figure 2).

\section{PDE4B and PDE4D protein levels in developing rat lung}

Subsequent studies focused on PDE4B and PDE4D variant protein expression. Using PDE4B-specific antibodies that recognized the common C-terminus of all PDE4B isoforms, a 
single immunoreactive signal was observed with an apparent molecular mass of $71 \mathrm{kDa}$ (Figure 3). The signal was faint at F17 and F19, increased slightly at F21 and was strong from P1 to P21. Using PDE4D-specific antibodies that recognized the common C-terminus of all PDE4D isoforms, four immunoreactive signals of 110, 105, 100, and 90-kDa, respectively, were detected with a differential pattern of expression during the developmental stages of the lungs. While the $110 \mathrm{kDa}$ signal decreased and was faint after birth, 105 and $100 \mathrm{kDa}$ signals were both more intense around P1 and P3, and the $90 \mathrm{kDa}$ signal was mainly observed after birth. Immunoreactive bands of short-form PDE4D variants were not detected in any of the developmental stages investigated.

\section{PDE4B2 and long-form PDE4D mRNA levels in lung during fetal and early postnatal}

life

Real-time PCR showed that PDE4B2 mRNA expression followed the same pattern as that of PDE4B detected with generic primers (Table 2). Among PDE4D long-form variants, PDE4D9 was the most abundant at F17, followed by PDE4D8, PDE4D3, PDE4D5, PDE4D4, and PDE4D7, and the most abundant form was expressed 15- to 16-fold higher than the least abundant form.

PDE4D3 mRNA levels increased 8-fold at birth and decreased at P7 (Figure 4 and Table 2). PDE4D4 mRNA fell sharply after F17, representing a 1,000-fold decrease, and remained low during all of the following stages of development. PDE4D5 increased sharply at birth, reaching 8-fold the F17 levels, and subsequently decreased to a level not statistically different from that observed at F17. PDE4D7 and PDE4D8 mRNA levels increased 15- and 7fold respectively from F17 to F21. Their levels remained high from F21 to P7 with a peak at P1 for PDE4D8. PDE4D9 mRNA levels increased 6-fold at birth as compared to F17 and decreased thereafter. 


\section{PDE4Ds localization during distal lung development}

Antibodies directed against PDE4B were not suitable for immunohistochemical assessment of their localization in the distal developing lung.

PDE4Ds were present at all stages of lung development investigated (F20, P1, P4, and P14) Before birth, immunolabeling was punctate and ubiquitous with no evidence of expression specificity in epithelial or mesenchymal cells (Figure 5); no pro-SP-C signal was detected at this stage. Labeling was the strongest at P1 when PDE4D proteins were mainly localized in the alveolar wall in thin, elongated cells with the morphologic appearance of alveolar type I cells as well as in mesenchymal cells. A few cells that stained for pro-SP-C, therefore likely to be alveolar type II cells, displayed some stains for PDE4D (Figure 6). At P4 and P14, the signal was very weak and mostly elongated (data not shown).. 


\section{Discussion}

The present study investigated the ontogeny of members of the PDE4 family during perinatal lung development in rat pups. Global PDE4 activity varied considerably during this period. This variation was associated with changes of the expression patterns of mostly PDE4B and PDE4D genes. In particular, PDE4D displayed a complex pattern with several isoforms being expressed at different phases of development. Immunofluorescence study of their localization in the distal lung suggested predominant expression in the walls of the alveoli with the most robust signal detected just after birth.

Previous studies have described changes in cAMP levels and protein kinase (PKA) activity during rat lung perinatal development (Whitsett et al., 1983; Acarregui et al., 1998). The only way to inactivate cAMP within cells is via hydrolysis by PDE, and among the 11 families of PDEs, PDE4 is thought to play a key role by modulating cAMP levels for many physiological events. The present study showed that global PDE4 activity increased towards the term of gestation, dropped at birth, then increased sharply to reach the highest level at the end of alveolarization. Global PDE4 activity in whole lung homogenates is the sum of the activities of several isoforms expressed from four genes, and only accounts for the total capacity of PDE4 enzymes to hydrolyze cAMP under the particular experimental conditions used and in the absence of external stimulation. However these variations of capacity to hydrolyze cAMP at time of key processes in lung development strongly suggest a role for PDE4s in both the preparation for air breathing and alveolarization.

It has been postulated that genes that regulate these processes should be differentially expressed during strictly defined period of times during perinatal life (Boucherat et al., 2007). Strategies based on longitudinal evaluation of gene expression in this period have identified significant changes in the expression of several genes involved in these processes (Clerch et al., 2004; Foster et al., 2006; Boucherat et al., 2007). This strategy was applied to the PDE4 
family by measuring steady-state levels of mRNAs for the four PDE4 genes, PDE4A-D, by real-time PCR. Because each gene expressed more than one variant, PDE4 mRNAs were initially examined by using generic primers allowing simultaneous amplification of all products of a particular gene. PDE4A mRNA steady-state levels were the highest among the various PDE4 in whole lung, and did not vary throughout the period investigated. PDE4C mRNA steady-state levels were the lowest of all PDE4 genes. Although PDE4C expression gradually increased during the first three weeks of life, this expression remained about 1,000fold lower than that of the other PDE4 genes at the end of alveolarization, suggesting that this gene did not play a major role in perinatal distal lung development. As measurements performed with whole lung tissue may hide strictly regulated expression in a few subsets of specialized cells, a role for these genes in perinatal lung development cannot be strictly excluded, but given the limited knowledge about the organization of the PDE4A and PDE4C variants and the absence of suitable biochemical tools to study them, the expression of these two genes was not investigated further.

Steady-state levels of PDE4B and PDE4D mRNAs presented the same pattern of expression: increasing just before birth, reaching levels at birth almost 30- to 60-fold higher than the levels at day 17 of fetal life, respectively, and slowly decreasing towards the end of alveolarization. This pattern infers global regulation of PDE4B and PDE4D expression during the perinatal period and suggests a role in lung development, especially shortly after birth. Western blot analysis of PDE4B and PDE4D expression was performed to provide further information on the expression of these two genes. Although some variants displayed similar migration on SDS-PAGE, this criterion allowed us to first circumvent some specific variants of these genes. Only one immunosignal was detected for the PDE4B gene with an apparent molecular weight of $71 \mathrm{kDa}$, which may correspond to the PDE4B2 variant, the only variant currently described to present this migration pattern in the rat (Iona et al., 1998). Real-time 
PCR measurements of PDE4B2 mRNA steady-state demonstrated an expression pattern similar to that obtained with generic pattern of PDE4B, implying that only PDE4B2 is expressed in the developing lung. The signal of this variant increased markedly from birth in terms of both mRNA and protein and remained strong at the protein level throughout the postnatal period, suggesting a role in adaptation to air breathing. PDE4B2 is expressed in several tissues with high expression in inflammatory cells (Wang et al., 1999). The invasion of macrophages into airspaces shortly after birth (van Rees et al., 1991) might account for this increase on the $1^{\text {st }}$ day of life but PDE4B2 expression may also be expressed in developing lung structures. Unfortunately, PDE4B antibodies available at the time of the study were not suitable to further localize this protein within distal lung (data not shown).

The expression pattern of PDE4D was much more complex during the perinatal period. Four apparent signals above $90 \mathrm{kDa}$ were observed in western blots that might represent the six long forms of PDE4D described to date, namely PDE4D3, PDED4, PDE4D5, PDE4D7, PDE4D8, and PDE4D9. According to the migration size of recombinant PDE4 in SDS-PAGE and the expression pattern of the mRNAs, the $110 \mathrm{kDa}$ signal may be PDE4D4, the $90 \mathrm{kDa}$ signals may be PDE4D3, PDE4D8, and PDE4D9, and the 100 and 105 kDa signals may be PDE4D5 and PDE4D7. Accordingly, the pattern of expression of these proteins reflected the pattern of expression of their respective mRNAs detected by quantitative PCR : PDE4D4 expression fell at P21, PDE4D3 (90 kDa), PDE4D5 (100 kDa), PDE4D8 (90 kDa), and PDE4D9 (90kDa) had a peak of expression at birth, that, however, dropped at P7 but remained significantly different from the expression at F20, at the exception of PDE4D5 expression. PDE4D4, PDE4D7, and PDE4D8 are selectively transcribed in a limited number of tissues, while PDE4D3, PDE4D5, and PDE4D9 are present in most tissues (Richter et al., 2005). PDE4D4 was absent in adult rat lung, consistent with its absence at the end of alveolarization. The fact that its expression was highest and fell sharply after F17 
suggested a role in late fetal development. The increase of PDE4D7 and PDE4D8 mRNAs just before birth and the relative stability of their expression during the alveolarization period, as well as their restricted expression within the organism suggest a more specific role in distal lung development. The relatively wide expression of PDE4D3, PDE4D5, and PDE4D9 within the organism infers they have a role in ubiquitous functional pathways shared by several tissues. These isoforms show a peak of expression at birth. Given the role of PDE4 in hormonal pathways (Mehats et al., 2002), one may speculate that at birth, hormonal regulations of postnatal lung physiology begin to be tightly regulated by selective PDE4s. Notably, clearance of fetal lung fluid is initiated secondary to beta-adrenergic-stimulated $\mathrm{Na}$ transport near term or at birth (Finley et al., 1998). Because PDE4D5 and PDE4D8 have been recently implicated in regulation of beta2-, and beta1-adrenergic signaling in ventricular cardiac myocytes (Richter et al., 2008), they might facilitate beta-adrenergic signaling after the surge of catecholamines during labor.

Attempts to localize PDE4D expression within specific cell types in the distal lung did not allow assignment of a selective variant to one particular cell type, but demonstrated robust expression in alveolar walls on the first day of life. The elongated appearance of staining suggested an anchored localization, consistent with a discrete function within alveolar cells. Although PDE4D isoforms are more abundant at P1, P3, P7 and P14 than at F20 in whole lung homogenates, the signals in the distal lung was faint at P4 and P14, suggesting a main site of PDE4D protein expression in the proximal lung and in bronchial tubes. Indeed PDE4D has been reported expressed in the smooth muscle of bronchi in adult lungs (Mehats et al., 2003). Further studies with antibodies directed against selective variants and manipulating expression tools in specific pulmonary epithelial and mesenchymal cells will help to decipher the role of these PDE4 in developing lungs. 
In conclusion, the present study identified complex patterns of expression of PDE4s in perinatal lung development. We have previously reported impaired alveolarization in rat pups chronically treated with rolipram (Mehats et al., 2008), and selective inhibition of discrete PDE4 isoforms could prevent this deleterious effect. Further investigations will be designed to characterize PDE4s functions. This might provide new therapeutic targets for the prevention and care of neonatal respiratory disorders. 


\section{Materials and methods}

\section{Reagents}

All reagents were from Sigma-Aldrich (L'Isle d'Abeau, France) unless otherwise indicated.

\section{Animals and lung tissue sampling}

Time-dated pregnant Sprague-Dawley rats were purchased from Charles River (SaintGermain sur l'Arbresle, France). Animal procedures complied with the rules of the Guide for Care and Use of Laboratory Animals and were approved by our Institutional Committee on Animal Use and Care. Lungs were collected between fetal day 17 and postnatal day 21. Rat pups were killed by an intraperitoneal overdose of sodium pentobarbital (70 mg/kg, Ceva, Libourne, France). Fetuses were retrieved by cesarean section from pentobarbital-anesthetized pregnant females. Lungs were immediately frozen in liquid nitrogen and kept at $-80^{\circ} \mathrm{C}$ until RNA extraction or biochemical analyses. For immunofluorescence studies, lungs were gently extracted, fixed through a polyethylene tracheal cannula with OCT Tissue Tek (Sakura Finetek, Zoeterwoude, The Netherlands) mixed with PBS, and stored at $-80^{\circ} \mathrm{C}$ until frozen sections were cut.

\section{RNA isolation and reverse transcription}

Total RNAs were extracted from frozen lung tissue or cell cultures using the RNeasy Plus Mini kit(Qiagen, Valencia, CA) according to the manufacturer's instructions. RNAs were reverse-transcribed into cDNA using $1 \mu \mathrm{g}$ of total RNA, Superscript III Reverse Transcriptase, and oligo-dT primers (Invitrogen, Cergy-Pontoise, France) according to the manufacturer's protocol. 


\section{PDE4 activity, mRNA expression, protein measurements and localization}

\section{PDE4 activity}

Lungs were homogenized in ice-cold hypotonic buffer (100 mM Tris-HCl pH 7.4), 2 $\mathrm{mM} \mathrm{MgSO}_{4}, 2 \mathrm{mM}$ EDTA, 50\% glycerol, and $1 \mathrm{mM}$ 2- $\beta$-mercaptoethanol supplemented with P2714 protease inhibitor cocktail, Sigma) using an all-glass homogenizer. Aliquots of homogenates were assayed for cAMP-PDE activity according to the method of Thompson and Appleman (Thompson et al., 1974). PDE activities were measured with $1 \mu \mathrm{M}$ acAMP as substrate (GE Healthcare, Amersham, Buckinghamshire, UK). PDE4 activity was defined as the fraction of cAMP PDE activity inhibited by $10 \mu \mathrm{M}$ rolipram. Protein concentrations were determined using the protein assay kit from Bio-Rad (Bio-Rad Laboratories, Hercules, CA) with BSA as standard.

\section{PDE4 mRNA expression level}

Similar amounts of the resulting first-strand cDNA were used as template for real-time PCR which was performed by Genoscreen (Lille, France). Primers were designed by Genoscreen and the primer sequences are given in Table 1 for the 4 genes and for the specific variants studied. Real-time PCR was performed on an ABI Prism 7000 device (Applied Biosystems) by Genoscreen. Relative expression was determined using the $\Delta \Delta \mathrm{Ct}$ (threshold cycle) method of normalized samples $(\Delta \mathrm{Ct})$, in relation to the expression of 18S RNA at F17 as a calibrator. This calibrator was identical throughout all PCR runs (Livak and Schmittgen, 2001).

\section{Measurement of PDE4 proteins by semiquantitative Western blot analysis}

Samples (30 $\mu \mathrm{g}$ protein/lane) were boiled in Laemmli buffer, subjected to electrophoresis on $10 \%$ SDS-PAGE, and blotted onto Hybond-P transfer membrane (GE Healthcare, Amersham). Membranes were blocked in 0.1\% Tris Buffered Saline-Tween 20 containing 5\% nonfat milk. Polyclonal rabbit anti-PDE4B antibody $(1: 2,500)$ (K118, kindly 
provided by Prof. Marco Conti (Iona et al., 1998)), and monoclonal mouse anti-PDE4D antibody (1:10,000) (61D10E, ICOS Corp., Seattle WA) were used (Bolger et al., 1997). Second-step HRP-conjugated antibodies $(1: 7,000)$ were purchased from Santa Cruz Biotechnology, Inc. (Santa Cruz, CA) and visualized by using ECL detection reagents (GE Healthcare Amersham). Labeling with beta-actin antibody (A2066, Sigma; 1:2,000) was used as a loading control. Densitometry data for band intensities in different sets of experiments was generated by analyzing the gel images on the Image $\mathbf{J}$ software (Version 1.33, USA).

\section{PDE4 localization by immunofluorescence and confocal microscopy}

Lungs fixed and stored as described above were cut into twenty-micrometer-thick tissue sections with a Jung CM3000 cryostat (Leica Microsystems GmbH, Wetzlar, Germany). Sections were fixed in $4 \%$ paraformaldehyde, permeabilized in $0.2 \%$ Triton, and saturated with $50 \mathrm{mM} \mathrm{NH}_{4} \mathrm{Cl}$. They were then incubated in $1 \%$ PBS-BSA-10\% donkey serum to block nonspecific binding. The primary antibody, a mouse monoclonal anti-PDE4D antibody (M3S1, provided by Prof. Marco Conti (Iona et al., 1998)) diluted to 1:65 in PBS was added and incubated overnight at $4^{\circ} \mathrm{C}$. The secondary antibody, an Alexa 488-conjugated donkey anti-mouse antibody (Molecular Probes, Invitrogen), diluted to 1:200, was added for 1 h. Sections were also incubated with an anti-pro-SP-C primary antibody (C-19, Santa Cruz Biotechnology), diluted to 1:200, then a Rhodamine-conjugated donkey anti-goat secondary antibody (TRITC: Jackson ImmunoResearch, Suffolk, UK), diluted to 1:200. After washing in PBS, nuclei were counter-stained with TOPRO 3 (Molecular Probes) diluted to 1:1000. Normal mouse IgG was used as negative control. Images were recorded on a Leica TCS SP2 confocal microscope (Leica Microsystems) equipped with a $\times 63$ oil-immersion objective (Numerical Aperture=1.32). The three channels were acquired sequentially with the following excitation and emission parameters: 488 nm, 500-540 nm for Alexa Fluor 488, 543 nm, 555- 
$615 \mathrm{~nm}$ for Rhodamine, and $633 \mathrm{~nm}, 645-750 \mathrm{~nm}$ for TOPRO-3. Gains were adjusted to avoid saturation pixel intensities. The three channels were color-coded, green, red, and blue, and merged.

\section{Statistical analysis}

Values are expressed as mean $\pm \mathrm{SE}$, unless otherwise indicated. Multiple comparisons of mean values were performed by nonparametric Kruskal-Wallis tests and two-group comparisons were performed by a Mann-Whitney U test. A value of $\mathrm{p}<0.05$ was considered to be significant. All calculations were performed with Graph Pad Prism software (San Diego, CA, USA). 


\section{Acknowledgments}

We are grateful to Josette Badet and Michelle Breuiller-Fouché for advice and assistance in immunofluorescence and to Nadine Segond and Bruno Saubamea for assistance in confocal microscopy. 


\section{References}

Acarregui MJ, Brown JJ, Penisten ST. 1998. Cyclic AMP-dependent protein kinase (PKA) gene expression is developmentally regulated in fetal lung. Biochim Biophys Acta 1402:303-312.

Aeberhard EE, Scott ML, Barrett CT, Kaplan SA. 1984. Effects of cyclic AMP analogues and phosphodiesterase inhibitors on phospholipid biosynthesis in fetal type II pneumocytes. Biochim Biophys Acta 803:29-38.

Bender AT, Beavo JA. 2006. Cyclic nucleotide phosphodiesterases: molecular regulation to clinical use. Pharmacol Rev 58:488-520.

Bolger GB, Erdogan S, Jones RE, Loughney K, Scotland G, Hoffmann R, Wilkinson I, Farrell C, Houslay MD. 1997. Characterization of five different proteins produced by alternatively spliced mRNAs from the human cAMP-specific phosphodiesterase PDE4D gene. Biochem J 328 ( Pt 2):539-548.

Boswell-Smith V, Spina D. 2007. PDE4 inhibitors as potential therapeutic agents in the treatment of COPD-focus on roflumilast. Int J Chron Obstruct Pulmon Dis 2:121-129.

Boucherat O, Franco-Montoya ML, Thibault C, Incitti R, Chailley-Heu B, Delacourt C, Bourbon JR. 2007. Gene expression profiling in lung fibroblasts reveals new players in alveolarization. Physiol Genomics 32:128-141.

Cardoso WV. 2001. Molecular regulation of lung development. Annu Rev Physiol 63:471494.

Clerch LB, Baras AS, Massaro GD, Hoffman EP, Massaro D. 2004. DNA microarray analysis of neonatal mouse lung connects regulation of KDR with dexamethasone-induced inhibition of alveolar formation. Am J Physiol Lung Cell Mol Physiol 286:L411-419. 
Conti M, Richter W, Mehats C, Livera G, Park JY, Jin C. 2003. Cyclic AMP-specific PDE4 phosphodiesterases as critical components of cyclic AMP signaling. J Biol Chem 278:54935496.

Finley N, Norlin A, Baines DL, Folkesson HG. 1998. Alveolar epithelial fluid clearance is mediated by endogenous catecholamines at birth in guinea pigs. J Clin Invest 101:972-981.

Fisher AB, Arad I, Dodia C, Chander A, Feinstein SI. 1991. cAMP increases synthesis of surfactant-associated protein A by perfused rat lung. Am J Physiol 260:L226-233.

Foster JJ, Goss KL, George CL, Bangsund PJ, Snyder JM. 2006. Galectin-1 in secondary alveolar septae of neonatal mouse lung. Am J Physiol Lung Cell Mol Physiol 291:L11421149.

Iona S, Cuomo M, Bushnik T, Naro F, Sette C, Hess M, Shelton ER, Conti M. 1998.

Characterization of the rolipram-sensitive, cyclic AMP-specific phosphodiesterases: identification and differential expression of immunologically distinct forms in the rat brain. Mol Pharmacol 53:23-32.

Livak KJ, Schmittgen TD. 2001. Analysis of relative gene expression data using real-time quantitative PCR and the 2(-Delta Delta C(T)) Method. Methods 25:402-408.

Mehats C, Andersen CB, Filopanti M, Jin SL, Conti M. 2002. Cyclic nucleotide phosphodiesterases and their role in endocrine cell signaling. Trends Endocrinol Metab 13:29-35.

Mehats C, Franco-Montoya ML, Boucherat O, Lopez E, Schmitz T, Zana E, Evain-Brion D, Bourbon J, Delacourt C, Jarreau PH. 2008. Effects of phosphodiesterase 4 inhibition on alveolarization and hyperoxia toxicity in newborn rats. PLoS ONE 3:e3445.

Mehats C, Jin SL, Wahlstrom J, Law E, Umetsu DT, Conti M. 2003. PDE4D plays a critical role in the control of airway smooth muscle contraction. Faseb J 17:1831-1841. 
Mendelson CR. 2000. Role of transcription factors in fetal lung development and surfactant protein gene expression. Annu Rev Physiol 62:875-915.

Odom MJ, Snyder JM, Mendelson CR. 1987. Adenosine 3',5'-monophosphate analogs and beta-adrenergic agonists induce the synthesis of the major surfactant apoprotein in human fetal lung in vitro. Endocrinology 121:1155-1163.

Richter W, Day P, Agrawal R, Bruss MD, Granier S, Wang YL, Rasmussen SG, Horner K, Wang P, Lei T, Patterson AJ, Kobilka B, Conti M. 2008. Signaling from beta1- and beta2adrenergic receptors is defined by differential interactions with PDE4. Embo J 27:384-393.

Richter W, Jin SL, Conti M. 2005. Splice variants of the cyclic nucleotide phosphodiesterase PDE4D are differentially expressed and regulated in rat tissue. Biochem J 388:803-811.

Spina D. 2008. PDE4 inhibitors: current status. Br J Pharmacol 155:308-315.

Thompson WJ, Brooker G, Appleman MM. 1974. Assay of cyclic nucleotide phosphodiesterases with radioactive substrates. Methods Enzymol 38:205-212.

van Rees EP, van der Ende MB, Sminia T. 1991. Ontogeny of macrophage subpopulations and Ia-positive dendritic cells in pulmonary tissue of the rat. Cell Tissue Res 263:367-373.

Wang P, Wu P, Ohleth KM, Egan RW, Billah MM. 1999. Phosphodiesterase 4B2 is the predominant phosphodiesterase species and undergoes differential regulation of gene expression in human monocytes and neutrophils. Mol Pharmacol 56:170-174.

Whitsett JA, Hull W, Dion C, Lessard J. 1985. cAMP dependent actin phosphorylation in developing rat lung and type II epithelial cells. Exp Lung Res 9:191-209.

Whitsett JA, Machulskis A, Noguchi A, Burdsall JA. 1982a. Ontogeny of alpha 1- and betaadrenergic receptors in rat lung. Life Sci 30:139-145.

Whitsett JA, Matz S, Darovec-Beckerman C. 1983. cAMP-dependent protein kinase and protein phosphorylation in developing rat lung. Pediatr Res 17:959-966. 
Whitsett JA, Noguchi A, Moore JJ. 1982b. Developmental aspects of alpha- and betaadrenergic receptors. Semin Perinatol 6:125-141. 


\section{Figure legends}

Figure 1. PDE4 activity in homogenates of whole lung from Sprague-Dawley rats at various fetal $(\mathrm{F})$ and postnatal $(\mathrm{P})$ ages. Values represent mean $\pm \mathrm{SD}$ on ten animals per group; nonparametric Kruskal-Wallis multiple-group comparison and 2-group comparisons by Mann-Whitney $U$-test. ${ }^{*} \mathrm{p}<0.05$ : significantly different from the $\mathrm{F} 17$ group; $*{ }^{*} \mathrm{p}<0.05$ : significantly different from the P1 group.

Figure 2. Real-time PCR analysis of PDE4A, PDE4B, PDE4C and PDE4D gene expression in developing rat lung at various fetal $(\mathrm{F})$ and postnatal $(\mathrm{P})$ ages. Values represent mean $\pm \mathrm{SD}$ on four animals per group, relative to mRNA levels at F17, arbitrarily set at 1 . Note that baseline values (see Table 2) are different for each gene. Baseline PDE4C was the lowest value, while PDE4D expression was about 64-fold higher and PDE4A and B expression was 1,000-fold higher. Nonparametric Kruskal-Wallis multiple-group comparison and 2-group comparisons by Mann-Whitney $U$-test: ${ }^{*} \mathrm{p}<0.05$ : significant different from the F17 group.

Figure 3. Western blots analysis of PDE4B and PDE4D protein levels in developing rat lung at various fetal $(\mathrm{F})$ and postnatal $(\mathrm{P})$ ages. Representative blots obtained with five normal rat lungs are shown. Beta-actin signal was used as protein loader control. Densitometric analyses of the five blots are plotted below the representative blots. Values represent mean \pm SD on five animals per group. Nonparametric Kruskal-Wallis multiple-group comparison and 2group comparisons by Mann-Whitney $U$-test: ${ }^{*} \mathrm{p}<0.05$ : significant different from the F17 group.

Figure 4. Real-time PCR analysis of PDE4D long form gene expression in developing rat lung at various fetal (F) and postnatal (P) ages. Values represent mean $\pm \mathrm{SD}$ on four animals 
per group, relative to mRNA levels at F17, arbitrarily set at 1 Nonparametric Kruskal-Wallis multiple-group comparison and 2-group comparisons by Mann-Whitney $U$-test: ${ }^{*} \mathrm{p}<0.05$ : significantly different from the F17 group.

Figure 5. Confocal microscopy images of PDE4D in the distal alveolar region of developing rat lung at F20. Immunofluorescence was performed using the PDE4D selective antibody M3S1 (labeled green, A). Nuclei were counterstained with TOPRO 3 (labeled blue, B). Bar = $25 \mu \mathrm{m}$ counterstaining mature type II alveolar epithelial cells, (C) merged image of A and B (B). Normal mouse IgG showed no signal (D). Representative panels for staining in two normal rat lungs at F20.

Figure 6. Confocal microscopy images of PDE4D in the distal alveolar region of developing rat lung at P1. Representative panels for staining in two normal rat lungs, (A) PDE4D selective antibody (labeled green), (B) pro-SP-C (labeled red), (C) Nuclei were counterstained with TOPRO 3 (labeled blue), (D) merged image. Bar $=37.5 \mu \mathrm{m}$. 
Tables

Table 1- Sequences of primers used in quantitative real-time PCR

\begin{tabular}{llll}
\hline Genes & Variants & Forward primer & Reverse primer \\
\hline 18S & & AAGTCCCTGCCCTTTGTACACA & GATCCGAGGGCCTCACTAAAC \\
PDE4A & Generic & CGTCAGTGCTGCGACAGTC & CCAGCGTACTCCGACACACA \\
PDE4B & Generic & GTGGCTGGGTACTCCCATAAT & GTCTGGCGTTGCTTCTTGGTA \\
& PDE4B2 & CCGTCAGTGGCGCCGGGAGC & CGAAATGTATTCAGACACTTGGT \\
PDE4C & Generic & CTCAGTCCGGGTCGAGTCT & AGGAGCGCCTGCCTAGTG \\
PDE4D & Generic & GCCAGCCTTCGAACTGTAA & TCTTGGAATGCAGGAATTGG \\
& PDE4D3 & GCATGTGAATACCTTCCCTTT & TGCACAAAGTTTGCTTGGAG \\
& PDE4D4 & CACGGAGCGCTACCTGTACT & TGCACAAAGTTTGCTTGGAG \\
& PDE4D5 & TGACCAGGAAAATGGCTCAG & CATTGTCCACATCGAAACATGTA \\
& PDE4D7 & GCCTCTGAGTGGATTACAGTTTC & CAGTCGAGTCTGCGGATGT \\
& PDE4D8 & TGGATCTTAGCCCTCGCTTT & TGCACAAAGTTTGCTTGGAG \\
& PDE4D9 & TATGAAGCCGAGATCCAGGT & TGCACAAAGTTTGCTTGGAG \\
& & & \\
\hline
\end{tabular}


Table 2- Analysis of PDE4 gene expression in whole lung tissue at various fetal (F) and postnatal $(\mathrm{P})$ ages: mean Delta $\mathrm{Ct}$

\begin{tabular}{cccccccc}
\hline & F17 & F19 & F21 & P1 & P7 & P14 & P21 \\
\hline PDE4A & $11.3 \pm 0.8$ & $11.4 \pm 1.5$ & $9.4 \pm 0.1$ & $9.5 \pm 1.4$ & $9.7 \pm 1.0$ & $10.1 \pm 0.5$ & $10.5 \pm 0.3$ \\
PDE4B & $11.3 \pm 0.6$ & $10.8 \pm 0.7$ & $8.0 \pm 0.4$ & $6.8 \pm 0.8$ & $8.5 \pm 1.2$ & $9.0 \pm 0.4$ & $10.8 \pm 0.4$ \\
PDE4C & $21.6 \pm 1.3$ & $20.3 \pm 1.3$ & $19.2 \pm 0.6$ & $18.4 \pm 0.7$ & $18.2 \pm 1.0$ & $17.0 \pm 0.6$ & $15.3 \pm 0.3$ \\
PDE4D & $14.8 \pm 1.0$ & $14.5 \pm 0.8$ & $10.9 \pm 0.6$ & $9.1 \pm 1.3$ & $8.7 \pm 1.5$ & $10.8 \pm 0.2$ & $12.0 \pm 0.4$ \\
\hline Isoforms & & & & & & & \\
\hline PDE4B2 & $12.6 \pm 0.6$ & $11.9 \pm 0.9$ & $9.1 \pm 0.3$ & $7.9 \pm 0.4$ & $9.4 \pm 1.2$ & $9.6 \pm 0.5$ & $11.5 \pm 0.4$ \\
PDE4D3 & $14.0 \pm 1.1$ & $15.2 \pm 0.6$ & $12.5 \pm 0.2$ & $11.1 \pm 0.6$ & $11.9 \pm 0.9$ & $12.8 \pm 0.1$ & $12.2 \pm 0.2$ \\
PDE4D4 & $15.2 \pm 0.0$ & $25.3 \pm 2.9$ & $21.6 \pm 3.9$ & $17.6 \pm 1.8$ & $17.7 \pm 3.7$ & $16.7 \pm 1.4$ & $18.7 \pm 1.1$ \\
PDE4D5 & $15.1 \pm 0.7$ & $16.9 \pm 1.4$ & $14.0 \pm 0.6$ & $12.1 \pm 0.6$ & $13.7 \pm 1.2$ & $14.8 \pm 0.2$ & $14.8 \pm 0.6$ \\
PDE4D7 & $15.9 \pm 0.9$ & $16.1 \pm 0.6$ & $11.8 \pm 0.4$ & $11.4 \pm 0.2$ & $11.7 \pm 1.4$ & $12.3 \pm 0.7$ & $11.9 \pm 0.6$ \\
PDE4D8 & $13.7 \pm 0.3$ & $13.2 \pm 0.6$ & $10.7 \pm 0.3$ & $10.1 \pm 0.7$ & $10.9 \pm 0.7$ & $11.6 \pm 0.3$ & $11.4 \pm 0.3$ \\
PDE4D9 & $11.5 \pm 0.6$ & $11.9 \pm 0.5$ & $10.6 \pm 0.7$ & $9.1 \pm 0.8$ & $9.4 \pm 0.8$ & $10.1 \pm 0.4$ & $10.5 \pm 0.2$ \\
\hline & & & & & & & \\
\hline
\end{tabular}

Data are presented as mean \pm SEM of data obtained from at least three animals at each age.

Delta Ct was calculated by subtracting Sample Ct to $18 \mathrm{~S}$ Ct of matching age. Note: a decrease in the Delta Ct value represents an increase in mRNA expression and a variation of 1 cycle means a two-fold increase or a decrease. 